\title{
Single-cell transcriptomics reveals the effect of PD-L1/TGF- $\beta$ blockade on the tumor microenvironment
}

\author{
Yoong Wearn Lim, Garry L. Coles, Savreet K. Sandhu, David S. Johnson, Adam S. Adler ${ }^{* \dagger}$ (D) and Erica L. Stone ${ }^{*+}$
}

\begin{abstract}
Background: The anti-tumor activity of anti-PD-1/PD-L1 therapies correlates with T cell infiltration in tumors. Thus, a major goal in oncology is to find strategies that enhance T cell infiltration and efficacy of anti-PD-1/PD-L1 therapy. TGF- $\beta$ has been shown to contribute to T cell exclusion, and anti-TGF- $\beta$ improves anti-PD-L1 efficacy in vivo. However, TGF- $\beta$ inhibition has frequently been shown to induce toxicity in the clinic, and the clinical efficacy of combination PD-L1 and TGF- $\beta$ blockade has not yet been proven. To identify strategies to overcome resistance to PD-L1 blockade, the transcriptional programs associated with PD-L1 and/or TGF- $\beta$ blockade in the tumor microenvironment should be further elucidated.

Results: We used single-cell RNA sequencing in a mouse model to characterize the transcriptomic effects of PD-L1 and/or TGF- $\beta$ blockade on nearly 30,000 single cells in the tumor and surrounding microenvironment. Combination treatment led to upregulation of immune response genes, including multiple chemokine genes such as CCL5, in macrophages, and downregulation of extracellular matrix genes in fibroblasts. Analysis of publicly available tumor transcriptome profiles showed that the chemokine CCL5 was strongly associated with immune cell infiltration in various human cancers. Further investigation with in vivo models showed that intratumorally administered CCL5 enhanced cytotoxic lymphocytes and the anti-tumor activity of anti-PD-L1.
\end{abstract}

Conclusions: Taken together, our data could be leveraged translationally to complement or find alternatives to anti-PD-L1 plus anti-TGF- $\beta$ combination therapy, for example through companion biomarkers, and/or to identify novel targets that could be modulated to overcome resistance.

Keywords: PD-L1, TGF- $\beta$, Tumor microenvironment, scRNA-seq, Immuno-oncology

\section{Background}

Antibodies blocking the PD-1/PD-L1 immune checkpoint pathway have been approved in the first-line setting for a range of cancer types including non-small-cell lung carcinoma (NSCLC), urothelial cancer, triple negative breast cancer, colorectal cancer, head and neck squamous cell carcinoma (HNSCC), microsatellite instability-high cancer, and melanoma. A major goal in oncology is to

\footnotetext{
* Correspondence: aadler@gigagen.com; estone@gigagen.com ${ }^{\dagger}$ Adam S. Adler and Erica L. Stone contributed equally to this work. GigaGen, Inc., One Tower Place, Suite 750, South San Francisco, CA 94080, USA
}

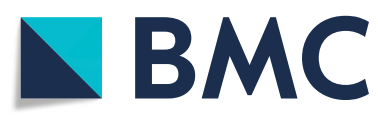

improve the response rate of these agents to benefit more patients. $\mathrm{T}$ cell excluded tumors are less likely to respond to immune checkpoint blockade targeting the PD-1/PDL1 pathway [1-3]. Furthermore, in metastatic urothelial cancer and some murine tumor models, $\mathrm{T}$ cell exclusion correlates with TGF- $\beta$ signaling, which is thought to induce expression of collagen and other factors leading to a physical barrier that prevents immune infiltration [3-5]. Consistently, in preclinical models, combination therapy with anti-PD-L1 plus anti-TGF- $\beta$ has been shown to induce $\mathrm{T}$ cell infiltration and synergistic anti-tumor efficacy $[3,5-10]$. However, TGF- $\beta$ blockade can lead to

(c) The Author(s). 2021 Open Access This article is licensed under a Creative Commons Attribution 4.0 International License, which permits use, sharing, adaptation, distribution and reproduction in any medium or format, as long as you give appropriate credit to the original author(s) and the source, provide a link to the Creative Commons licence, and indicate if changes were made. The images or other third party material in this article are included in the article's Creative Commons licence, unless indicated otherwise in a credit line to the material. If material is not included in the article's Creative Commons licence and your intended use is not permitted by statutory regulation or exceeds the permitted use, you will need to obtain permission directly from the copyright holder. To view a copy of this licence, visit http://creativecommons.org/licenses/by/4.0/ The Creative Commons Public Domain Dedication waiver (http://creativecommons.org/publicdomain/zero/1.0/) applies to the data made available in this article, unless otherwise stated in a credit line to the data. 
significant toxicity, which has led to termination of several clinical trials testing TGF- $\beta$ /TGF- $\beta$ RII inhibitors $[11,12]$, and clinical efficacy has not yet been demonstrated conclusively. Deeper understanding of the transcriptional programs involved in anti-PD-L1 plus anti-TGF- $\beta$ combination therapy could lead to improved approaches that address these shortcomings.

The transcriptional programs induced by PD-L1 plus TGF- $\beta$ signaling blockade remain poorly understood. Single-cell RNA sequencing (scRNA-seq) is a powerful tool that provides transcriptional profiles of tens of thousands of cells, enabling comprehensive analysis of the tumor microenvironment. Thus, we used scRNA-seq to profile the transcriptional changes induced at the single-cell level in the tumor microenvironment of a mouse model after combination anti-PD-L1 plus antiTGF- $\beta$ treatment. In particular, we wanted to identify genes that are altered during a productive anti-tumor immune response that may enhance $\mathrm{T}$ cell infiltration and efficacy of PD-1/PD-L1 blockade, with the ultimate goal of discovering novel therapeutic strategies to overcome resistance to $\mathrm{PD}-1 / \mathrm{PD}-\mathrm{L} 1$ blockade.

We found that anti-PD-L1 (atezolizumab) plus antiTGF- $\beta$ led to reduced expression of collagen and other matrix remodeling genes by cancer-associated fibroblasts. Anti-PD-L1 induced the expression of several chemokines that are associated with the recruitment of cytotoxic $\mathrm{T}$ cells, with a further increase in expression after combination therapy. Analysis of The Cancer Genome Atlas (TCGA) transcriptome data confirmed that multiple chemokines are associated with immune cell infiltration in human tumors, and revealed C-C Motif Chemokine Ligand 5 (CCL5) as the chemokine most highly correlated with immune infiltration across several tumor types. Finally, intratumoral administration of CCL5 increased the frequency of CCR5 + CD8+ T cells and mature $\mathrm{CD} 11 \mathrm{~b}+\mathrm{NK}$ cells within the tumor, and administration of CCL5 plus anti-PD-L1 (atezolizumab) induced tumor growth inhibition over anti-PD-L1 alone in the murine colon tumor model MC38. Taken together, our data could be leveraged translationally to improve anti-PD-L1 plus anti-TGF- $\beta$ combination therapy, for example through companion biomarkers, and/or to identify novel targets that could be modulated to overcome resistance.

\section{Results}

PD-L1 plus TGF- $\beta$ blockade reduces tumor growth and enhances immune cell infiltration

Anti-TGF- $\beta$ has been shown to enhance the efficacy of PD-L1 blockade in several murine models $[3,5,6,8,13$, 14]. To confirm these results, and to investigate whether anti-TGF- $\beta$ enhances the anti-tumor efficacy of the FDA-approved anti-PD-L1 antibody atezolizumab, human
PD-L1 knock-in mice bearing subcutaneous (s.c.) colon carcinoma MC38 tumors expressing Hu-PD-L1 (Hu-PDL1-MC38) were treated with vehicle, anti-PD-L1, or a combination of anti-PD-L1 plus anti-TGF- $\beta$. As expected, anti-PD-L1 significantly limited tumor growth $(p=$ 0.0087; Fig. 1a, b), while anti-TGF- $\beta$ alone was not efficacious (Additional file 1, Figure S1a, b). Combination treatment of anti-PD-L1 plus anti-TGF- $\beta$ was significantly more efficacious than anti-PD-L1 alone $(p=0.038)$ and led to tumor regression in four of six $(66.67 \%)$ animals by day 28 (Fig. 1a, b). Both anti-PD-L1 and anti-PD-L1 plus anti-TGF- $\beta$ also improved survival relative to control (Fig. 1c). One of six (16.67\%) mice from the anti-PD-L1 group and three of six (50\%) mice from the anti-PD-L1 plus anti-TGF- $\beta$ group had a complete response and were rechallenged with Hu-PD-L1 MC38 cells, implanted s.c. on the opposite flank from the original tumor. Tumor-naïve, wildtype C57BL/6 mice were used as controls. All previously cured mice, but not the naïve mice, were protected from tumor re-challenge, indicating the presence of antitumor immune memory ( $p=0.026$; Additional file 1 , Figure S1c). To investigate if PD-L1 plus TGF- $\beta$ blockade had an effect on $\mathrm{T}$ cell infiltration, CD3 immunohistochemistry (IHC) was performed on tumors from the treated mice. Anti-PD-L1 plus anti-TGF- $\beta$ significantly increased $\mathrm{T}$ cell infiltration while anti-PD-L1 alone did not (Fig. 1d). In a different mouse colon carcinoma tumor model, CT26, significant tumor growth inhibition was also observed upon anti-PD-L1 (atezolizumab, which cross reacts with murine PD-L1) plus anti-TGF- $\beta$ treatment, whether anti-TGF- $\beta$ was given intraperitoneally (I.P.) or intratumorally (I.T.) (Additional file 1, Figure S2).

To study this therapeutic regimen in an orthotopic tumor type, we treated mice harboring EMT6 breast tumors orthotopically grown in the mammary fat pad with PBS, anti-PD-L1 (atezolizumab), anti-TGF- $\beta$, or anti-PD-L1 (atezolizumab) plus anti-TGF- $\beta$. While we did not observe efficacy from either single agent, antiPD-L1 plus anti-TGF- $\beta$ significantly reduced tumor size relative to PBS or anti-TGF- $\beta$ alone $(p<0.03$; Fig. $1 \mathrm{e}, \mathrm{f})$. Further, the combination treatment improved the survival relative to all individual arms $(p<0.01$; Fig. $1 \mathrm{~g})$. Anti-PD-L1 plus anti-TGF- $\beta$ in combination, but neither monotherapy alone, enhanced CD3 immune cell infiltration relative to all individual arms ( $p<0.03$; Fig. 1h).

Together, these results established that dual blockade of PD-L1 and TGF- $\beta$ effectively controlled tumor growth and improved $\mathrm{T}$ cell infiltration into the tumor in multiple mouse models. We thus reasoned that in vivo inhibition of PD-L1 plus TGF- $\beta$ could be used as an experimental model to identify genes important for immune cell infiltration and anti-tumor response, and this information could be used to uncover strategies to overcome resistance to anti-PD-L1 therapy. The EMT6 





(See figure on previous page.)

Fig. 1 Anti-PD-L1 plus anti-TGF- $\beta$ leads to tumor regression in mice. a-d Hu-PD-L1 Kl mice bearing s.c. Hu-PD-L1-MC38 tumors ( $n=6$ per group) were dosed I.P. biweekly for 3 weeks with PBS, aPD-L1 (2 mg/kg; atezolizumab), or aPD-L1 plus aTGF- $\beta$ (10 mg/kg; 1D1 1). a Average MC38 tumor volume \pm SEM is shown. $P$ values were determined using Wilcoxon rank sum test, comparing tumor sizes on day 18. b Spider plots showing MC38 tumor volume for individual mice over time. $\mathbf{c}$ Survival plot for the MC38 study. $P$ values were determined using log-rank test. $\mathbf{d}$ CD3 IHC score in MC38 tumors by immunohistochemistry (IHC). Each point is the IHC score representing the density of CD3+ cells from an individual mouse. The horizontal black bars of the box plots indicate median score, while the lower and upper hinges correspond to the first and third quartiles, respectively. $P$ values were determined using Wilcoxon rank sum test. $\mathbf{e}-\mathbf{h}$ Mice bearing orthotopic EMT6 tumors were treated with PBS, aPD-L1 (10 mg/kg for the first dose with each subsequent dose at $5 \mathrm{mg} / \mathrm{kg}$; atezolizumab), aTGF- $\beta$ (10 mg/kg; 1D11), or aPD-L1 plus aTGF- $\beta$. For all treatments, the first dose was administered I.V. on day 0 and the eight subsequent doses were administered I.P. three times per week. $n=12$ mice per group, including 3 mice per group taken down early on day 8 for scRNA-seq analysis. e Average EMT6 tumor volume \pm SEM is shown. $P$ values were determined using Wilcoxon rank sum test. Mice removed early for scRNA-seq analysis were not included in the average. $\mathbf{f}$ Spider plots showing EMT6 tumor volume for individual mice over time. Mice sacrificed for scRNA-seq ( $n=3$ per group) are shown in red. $\mathbf{g}$ Survival plot for the EMT6 study. $P$ values were determined using log-rank test. $\mathbf{h}$ CD3 IHC score in EMT6 tumors. Each point is the IHC score representing the density of CD3+ cells from an individual mouse. The horizontal black bars of the box plots indicate median score, while the lower and upper hinges correspond to the first and third quartiles, respectively. $P$ values were determined using Wilcoxon rank sum test. Non-significant $p$ values are not shown for all panels

orthotopic tumor model provided us with an opportunity to characterize the single-cell molecular responses to anti-PD-L1 \pm anti-TGF- $\beta$ treatment in a setting where anti-TGF- $\beta$ addition overcomes resistance to anti-PD-L1 therapy. Eight days after the first dose, three representative tumors per group were harvested (Fig. 1f, in red), dissociated, flow sorted for CD45+ immune cells and CD45- non-immune cells, and subjected to scRNA-seq.

\section{Single-cell RNA sequencing of tumors from anti-PD-L1 \pm anti-TGF- $\beta$-treated mice}

After quality control (Additional file 1, Figure S3), single-cell transcriptomic profiles for 27,460 high-quality cells were generated, including 17,665 CD45+ and 9795 CD45- cells. Uniform Manifold Approximation and Projection (UMAP) dimensionality reduction of the transcriptomes revealed distinct clusters of cells present in all treatment groups (Fig. 2a, b). We assigned cell type labels using SingleR, which annotated cell types based on reference transcriptomes of pure cell types in the ImmGen database [15]. To discern tumor cells from host stromal cells, we further performed fine UMAP clustering on the CD45- cells, generating 15 distinct clusters (Additional file 1, Figure S4a). Cells in cluster 7 had high expression of the fibroblast marker Fap and were thus annotated as fibroblasts (Additional file 1, Figure S4b). The remaining cells were annotated as tumor cells or assigned their original SingleR labels as endothelial or epithelial cells. Copy number analysis confirmed that the annotated tumor cells exhibited aberrant copy number while the fibroblasts did not (Additional file 1, Figure S4c). In total, we identified 12 cells types that had both unique and overlapping marker gene expression patterns (Additional file 1, Figure S5). When comparing the treatment groups, we observed differences in cell type composition. For example, the anti-PD-L1, antiTGF- $\beta$, and anti-PD-L1 plus anti-TGF- $\beta$ samples had lower numbers of macrophages $(9.2-20.5 \%$ of CD $45+$ cells) compared to the PBS control (33.3\% of CD45+ cells; Additional file 1, Figure S6a). Conversely, relative to the PBS sample where B cells represented $9.5 \%$ of the $\mathrm{CD} 45+$ cells, the three treatment groups had higher numbers of B cells (23.8-32.1\%).

Next, we performed differential gene expression analysis between each drug treatment group and the control sample, within each annotated cell type. Anti-PD-L1, anti-TGF- $\beta$, and anti-PD-L1 plus anti-TGF- $\beta$ treatments resulted in 568, 123, and 358 differentially expressed genes across different cell types, respectively (Additional file 2, Table S1). Because multiple tumors were pooled for each treatment group for the scRNA-seq analysis, it is possible that some of the gene expression differences observed could be skewed by a single tumor sample, leading to a higher number of false positives from this analysis. Among CD45- cells, the combo treatment resulted in the highest number of downregulated genes in fibroblasts (Fig. 2c). To further investigate the changes in fibroblasts, we performed unsupervised re-clustering of fibroblasts, revealing two subclusters. Cluster Fib_0 was associated with higher expression of genes including Clec3b, Tnxb, Col14a1, Pi16, and Lbp, while cluster Fib_ 1 was associated with higher expression of Tnc, Gapdh, Spp1, Cxcl14, and Timp1 (Fig. 2d-f). We performed differential gene expression and functional enrichment analysis within the fibroblast subclusters. Interestingly, within Fib_0, dual blockade of PD-L1 plus TGF- $\beta$ led to significant downregulation of matrix remodeling associated functional categories including collagen fibril organization, extracellular matrix organization, and fibrillar collagen trimer (Fig. 2g). For example, collagen and metalloproteinase genes such as Col1a2, Col5a2, Col1a1, P3h4, Cthrc1, and $M m p 2$ were downregulated in Fib_0 following combination blockade (Fig. 2h, i; Additional file 2, Table S2). This is consistent with previous observations suggesting that TGF- $\beta$ blockade synergizes with antiPD-L1 to reprogram the peritumoral stromal fibroblasts [3]. 


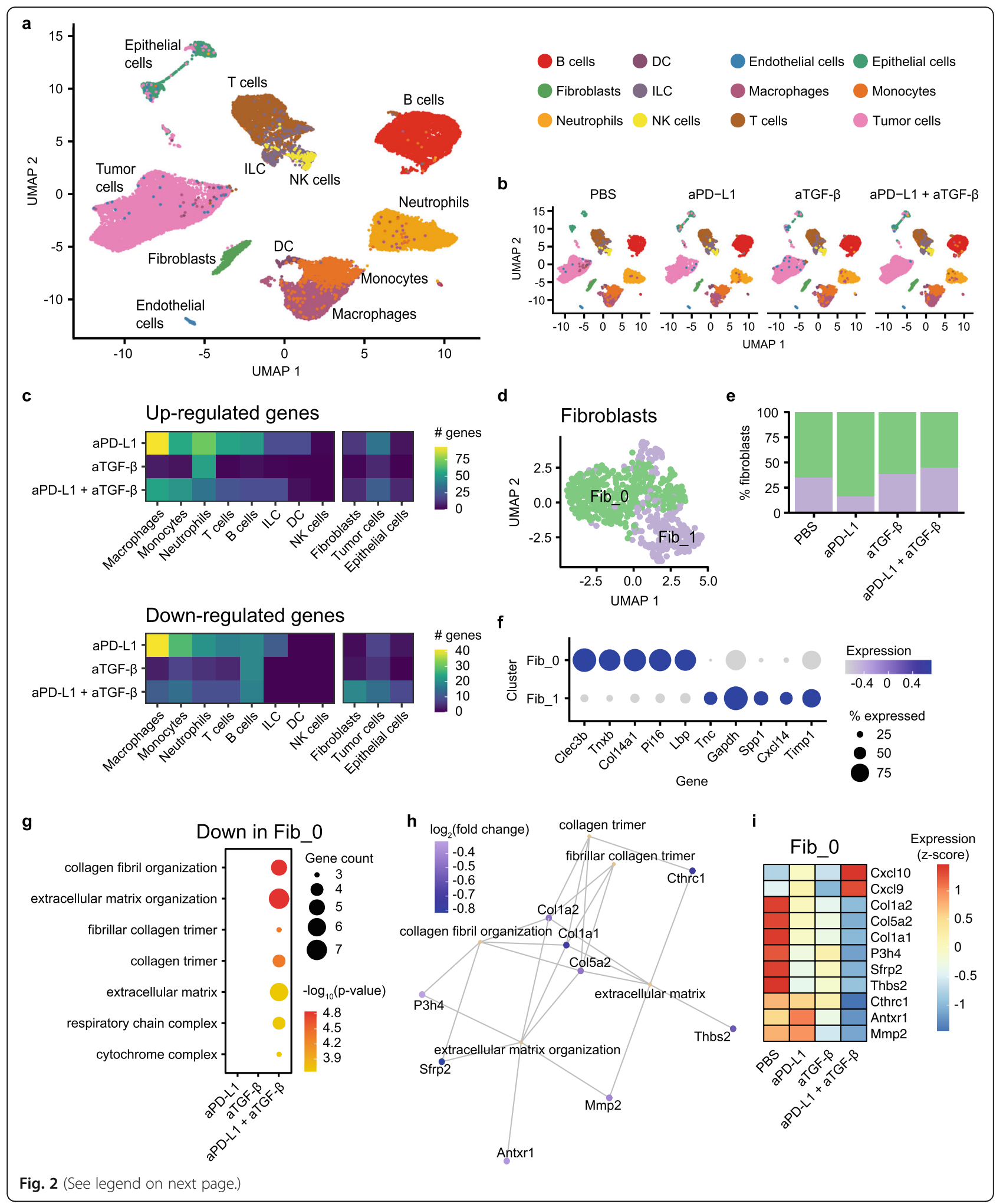




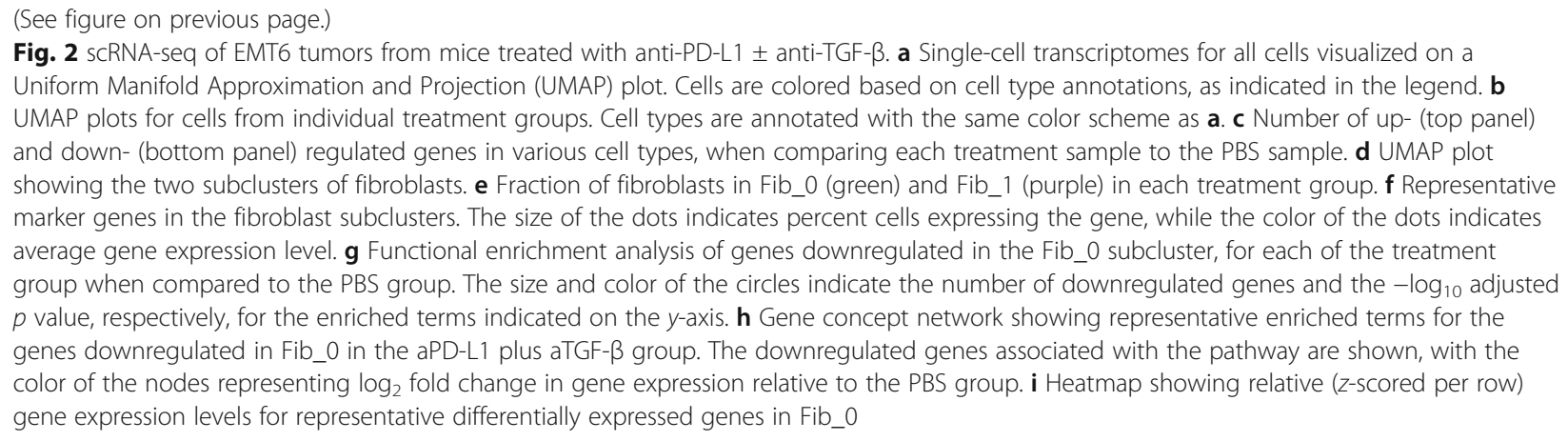

Among CD45+ cell types, macrophages had the highest number of upregulated genes following anti-PD-L1 plus anti-TGF- $\beta$ treatment (Fig. 2c). Fine clustering of macrophages revealed four subclusters, with subclusters Mac_0 and Mac_1 representing more than $90 \%$ of the cells (Fig. 3a, b). Cells in Mac_0 were characterized by expression of complement-related genes (C1qa, C1qb, C1qc) and cytokines (Spp1, Ccl12), while cells in Mac_1 expressed higher levels of several tumor-associated macrophage-related genes such as Arg1, Cebpb, and Ier3 (Fig. 3c). Differential expression and functional enrichment analysis showed that anti-PD-L1, administered alone or in combination with anti-TGF- $\beta$, resulted in enhanced immune responses in Mac_0. For example, both anti-PD-L1 and anti-PD-L1 plus anti-TGF- $\beta$ induced upregulation of genes involved in antigen processing and presentation, adaptive immune response, response to bacterium, and response to interferongamma (Fig. 3d, e). Anti-TGF- $\beta$, administered alone or in combination with anti-PD-L1, induced upregulation of genes involved in monocyte chemotaxis, response to chemokine, and eosinophil migration (Fig. 3d). Notably, many chemokines were upregulated in the Mac_0 population of the anti-PD-L1 sample $(\mathrm{Ccl}$, Cxcl9, Ccl4) and the anti-TGF- $\beta$ sample $(C c l 2, C c l 12, C c l 4)$, while multiple chemokines showed further upregulation after anti-PD-L1 plus anti-TGF- $\beta$ combination treatment (Ccl5, Ccl2, Ccl7, Ccl3, Cxcl10) (Fig. 3f; Additional file 2, Table S3). This is consistent with previous in vivo studies showing anti-PD-1/PD-L1 treatment favors polarization of macrophages towards a more immunostimulatory state $[16,17]$.

Further analysis revealed that chemokines were upregulated in fibroblasts, neutrophils, and T cells in anti-PDL1 and anti-PD-L1 plus anti-TGF- $\beta$-treated tumors (Additional file 1, Figure S6b). Additionally, anti-PD-L1 plus anti-TGF- $\beta$ combination treatment led to upregulation of additional chemokines such as Cxcl1 and Cxcl2 in tumor cells above and beyond those induced by either single treatment alone (Additional file 2, Table S1). Chemokines lay a critical role in recruiting various immune cells to create an inflamed tumor microenvironment $[18,19]$. The enhanced chemokine expression in samples after anti-PD-L1 plus anti-TGF- $\beta$ combination treatment suggests that chemokines may facilitate the success of checkpoint blockade treatments. Indeed, both pre- and post-treatment CXCL9 and CXCL1O expression levels correlate with response to anti-PD-1 treatment in melanoma patients and anti-PD-L1 treatment in urothelial cancer patients [20-22].

A major goal in immunotherapy is to find strategies to enhance immune cell infiltration. While TGF- $\beta$ monoclonal antibodies in combination with anti-PD-L1s have been shown to enhance immune infiltration and efficacy in preclinical models, TGF- $\beta$ targeting molecules have been plagued by toxicity, leading to the halting of several clinical trials with these agents. As an alternative, our data suggest the possibility that a chemokine administered therapeutically may improve immune cell infiltration and efficacy in combination with anti-PD-L1 antibodies.

\section{CCL5 expression is associated with immune cell infiltration across human cancer types}

The scRNA-seq data suggested that the enhanced expression of chemokines in tumors treated with antiPD-L1 plus anti-TGF- $\beta$ may contribute to the ability of anti-TGF- $\beta$ to enhance the anti-tumor efficacy of antiPD-L1. To further investigate the chemokines that may be most important for enhancing efficacy of anti-PD-L1 therapy, we took a computational approach to identify chemokines associated with immune cell infiltration in different human cancer types using The Cancer Genome Atlas (TCGA). For this we utilized tumor-infiltrating lymphocyte (TIL) scores for TCGA tumor samples, which have been previously quantified using two distinct methods. For one method, Saltz et al. used a deep learning image recognition algorithm to quantify TILs from hematoxylin and eosin (H\&E)-stained pathology images for 13 TCGA cancer types [23, 24]. Independently, Aran et al. developed a gene signature-based method termed xCell to infer immune cell abundance based on tumor gene expression profiles [25]. Specifically, we focused on 


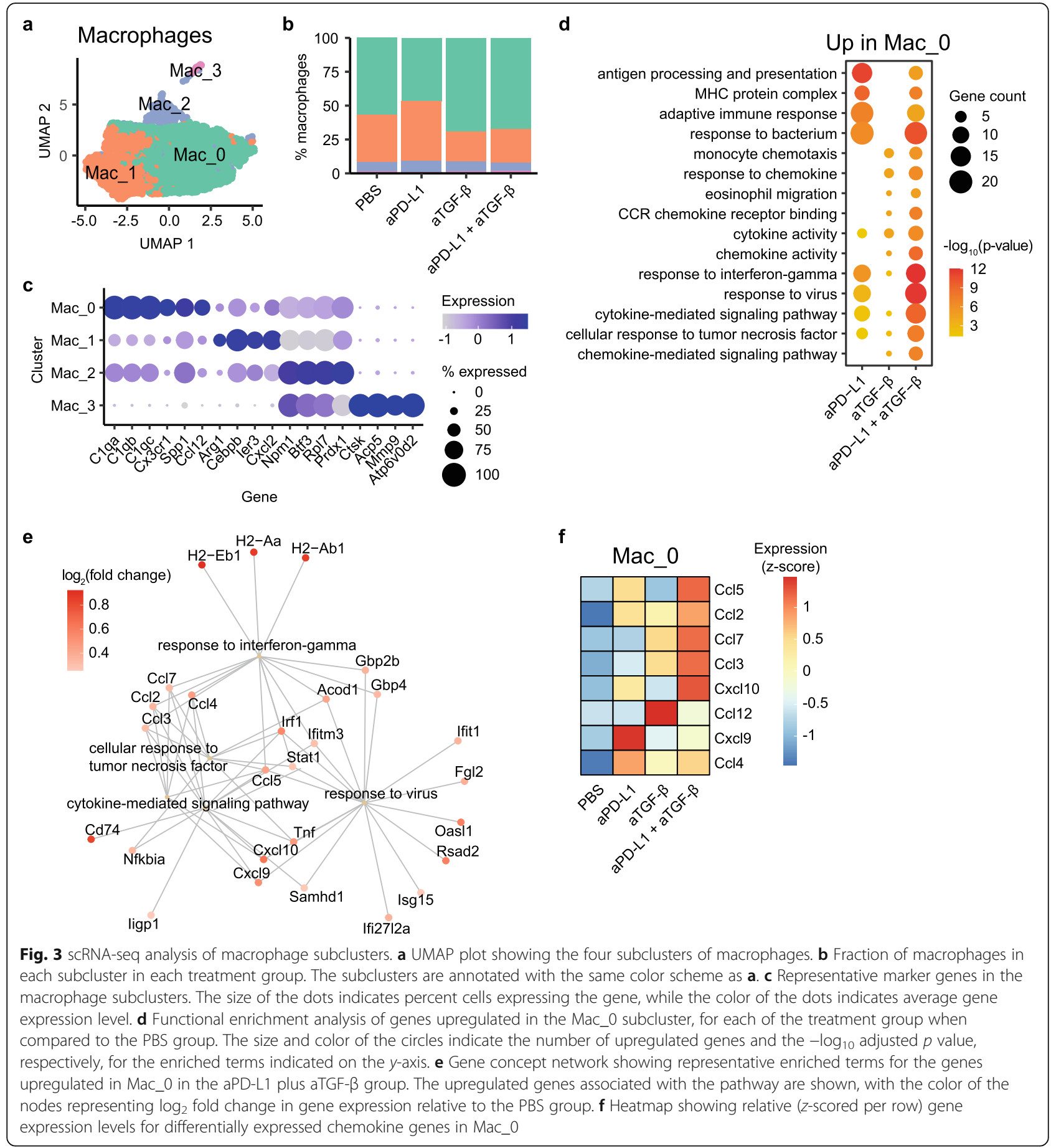

the immune cell abundance scores for three cytotoxicity relevant cell types: CD8+ T cells, dendritic cells, and NK cells. Using linear regression models, we tested for association between gene expression levels for a panel of chemokine genes (i.e., genes starting with CXC, CCL, $\mathrm{CX} 3 \mathrm{C}$, or $\mathrm{XC}$ ) with both the image-based and gene signature-based immune infiltration scoring methods (Fig. 4a). Chemokines significantly associated with immune infiltration scores were further ranked based on their association strengths (i.e., regression coefficients) across cancer types (multiple testing adjusted $p$ values $<0.05$ ).

The expression levels of six chemokine genes (CCL5, CXCL9, CXCL13, CXCL10, CXCL11, and CCL19) were significantly associated with the image-based immune scores across all 13 tested cancer types (Fig. 4b). For example, correlation of CCL5 expression in breast cancer 
a

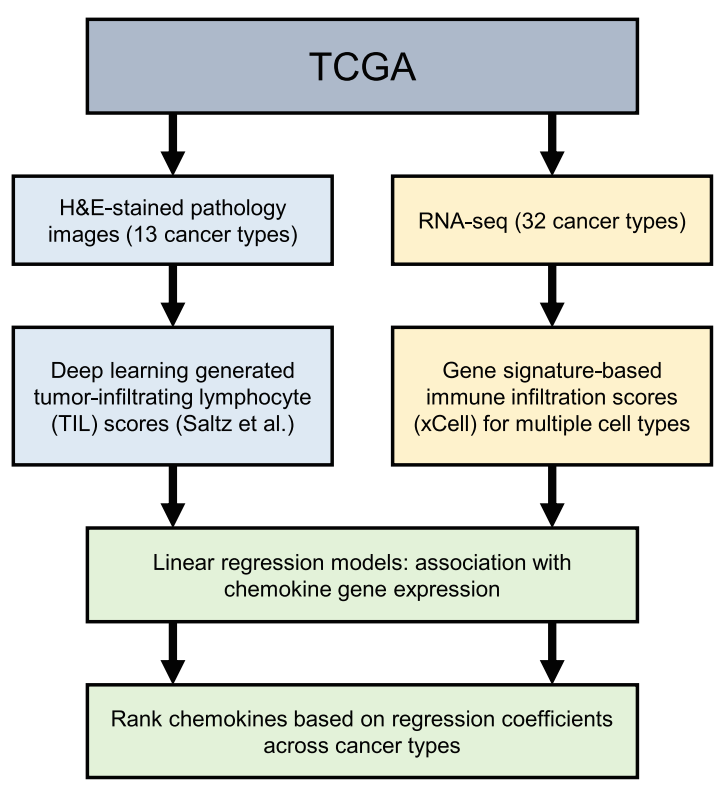

b

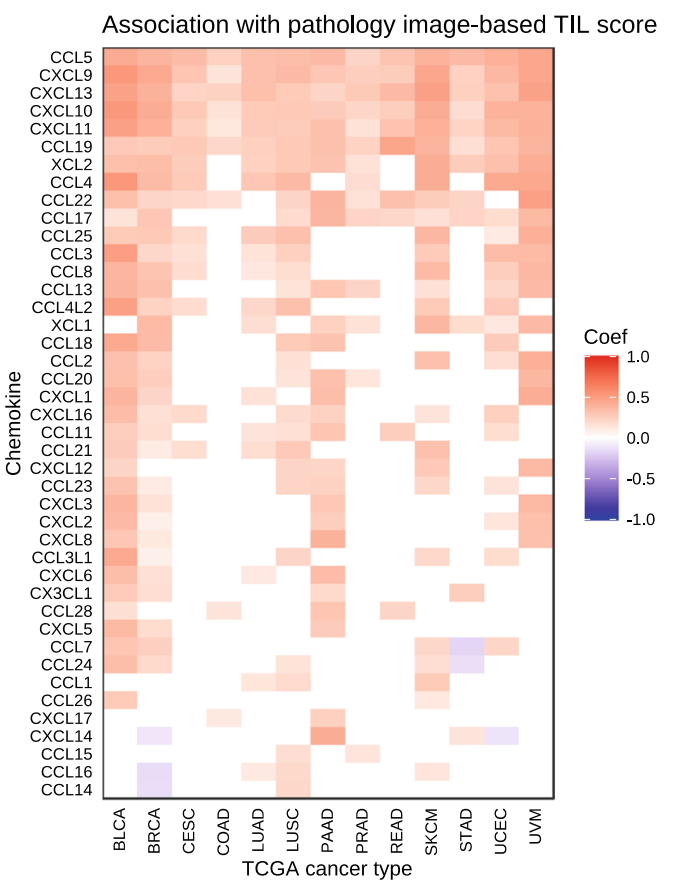

C

Association with gene signature-based score
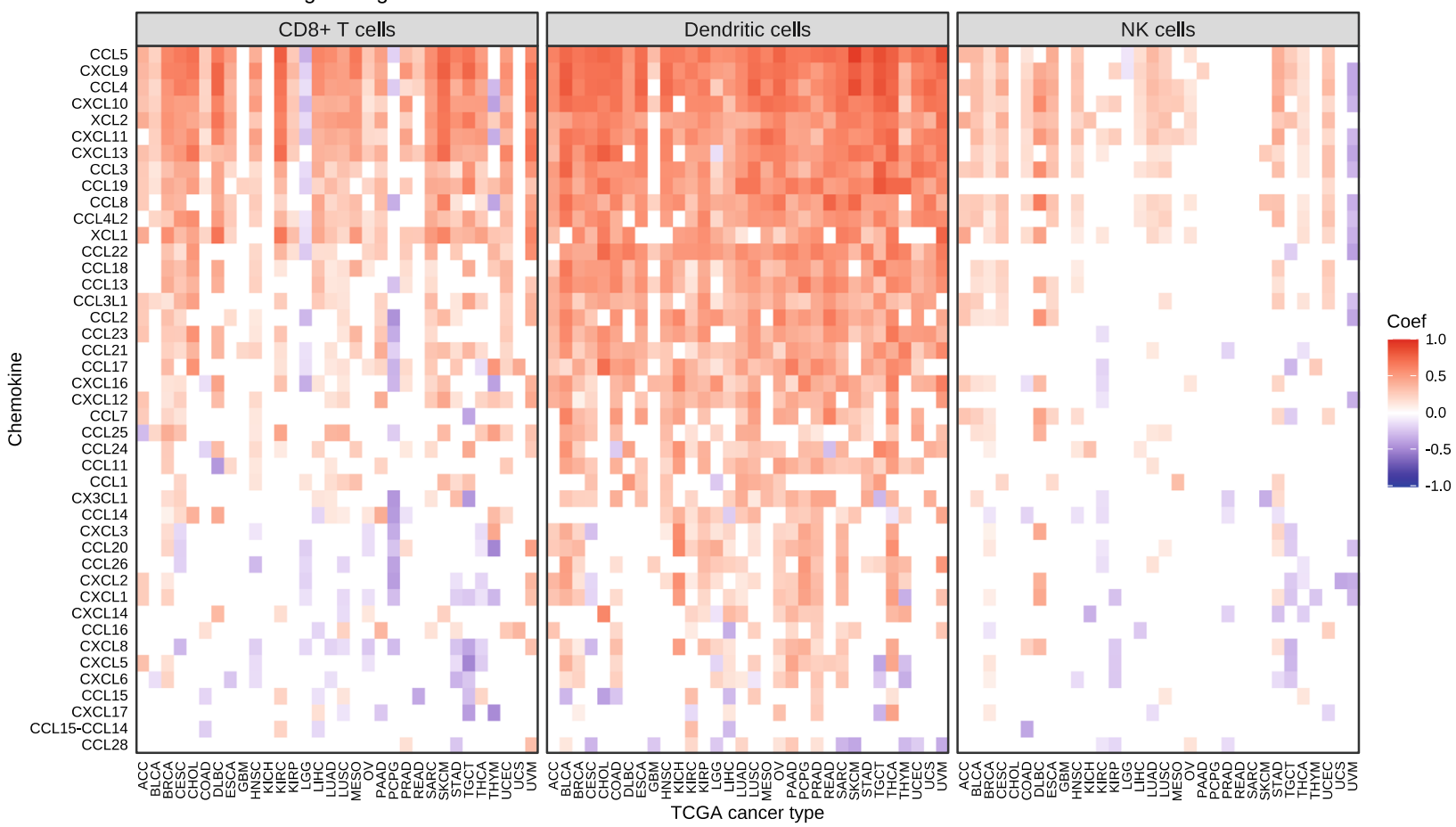

Fig. 4 Association of chemokine gene expression with inferred immune cell infiltration in TCGA. a Analysis workflow to identify chemokine genes associated with immune cell abundance in TCGA tumor samples. Two parallel methods were used to infer immune cell abundance: image-based estimation from pathology slides (left) and gene signature-based estimation from RNA-seq (right). Linear regression models were used to find chemokine genes whose expression levels are associated with inferred immune infiltration. $\mathbf{b}$ Heatmap showing chemokines (rows) significantly associated with the pathology image-based TIL scores across different TCGA cancer types (columns). The color indicates regression coefficients from the linear models (red/orange = positive association between chemokine expression and immune infiltration). $\mathbf{c}$ Heatmap showing chemokines (rows) significantly associated with the gene signature-based immune scores across different TCGA cancer types (columns). The panels represent associations with CD8+ T cells, dendritic cells, and NK cell scores. The chemokines (rows) are ordered descendingly by the sum of the regression coefficients across all cancer types and all 3 immune cell types (i.e., all columns) 
(BRCA) with the image-based infiltration scores is shown in Figure S7a (Additional file 1). CCL5, CXCL9, CXCL13, CXCL10, and CXCL11 were also among the top chemokine genes associated with the gene expression-based immune scores across multiple cancer types (Fig. 4c). Conversely, these chemokines were weakly associated with the gene expression-based immune scores for immunosuppressive Tregs and M2 macrophages (Additional file 1, Figure S7b). Notably, CCL5 was consistently the top chemokine associated with immune infiltration scores, as determined by both the image-based and the gene signature-based methods. CCL5 expression levels were significantly associated with inferred CD8 + T cell, dendritic cell, and NK cell abundance across multiple cancer types (Fig. 4c). These findings are consistent with the chemotactic roles of CCL5 in regulating immune cell trafficking in tumors via interactions with its receptor CCR5 [26].

\section{Combination therapy of anti-PD-L1 plus intratumorally administered CCL5 leads to tumor growth inhibition}

Given its chemotactic properties, we hypothesized that intratumoral delivery of recombinant CCL5 protein may enhance immune cell recruitment and control tumor growth. While multiple intratumoral cell subsets express CCR5 (Fig. 5a; Additional file 1, Figure S8) [27, 28], intratumorally administering recombinant CCL5 to s.c. MC38 tumors resulted in an increased frequency of CD8+ $\mathrm{T}$ cells in the tumor that were CCR5 positive 12 days after starting dosing ( $p=0.03$; Fig. $5 \mathrm{a}$, b; Additional file 1, Figure S8), though no significant difference was seen for total CD8+ $\mathrm{T}$ cells (Fig. 5a). There was also a trend towards an increase in the frequency of NK cells expressing CCR5 ( $p=0.09$; Fig. 5a, b; Additional file 1, Figure S8) but not for total NK cells (Fig. 5a). Furthermore, we found that intratumoral CCL5 resulted in an increased frequency of NK cells expressing CD11b ( $p=$ 0.04; Fig. 5c, d; Additional file 1, Figure S8). Though we note that after multiple hypothesis correction (Bonferroni), none of the $p$ values in Fig. 5b or d remain significant $(p>0.05)$. CD11b expression by NK cells has been reported to mark mature NK cells with enhanced effector function [29]. This increase in mature NK cells may be a result of the ability of CCL5 to activate NK cells and/or recruit activated NK cells [30]. Together, these data suggest that intratumorally administered CCL5 altered the tumor microenvironment, including by recruiting cytotoxic lymphocytes.

Lastly, we determined if intratumorally administered CCL5 was able to enhance the efficacy of anti-PD-L1 therapy using the same tumor model. While neither intratumoral CCL5 nor anti-PD-L1 (atezolizumab) alone inhibited tumor growth, CCL5 in combination with antiPD-L1 significantly reduced tumor growth relative to all individual arms $(p<0.05$; Fig. 5e, f). Mice that received CCL5 plus anti-PD-L1 combination therapy also had significantly prolonged survival compared to all individual arms $(p<0.02$; Fig. 5g). Thus, intratumorally administered CCL5 is able to overcome resistance to anti-PD-L1 therapy.

\section{Discussion}

For the first time, we used scRNA-seq to investigate gene expression changes in tens of thousands of single cells associated with anti-PD-L1 and/or anti-TGF- $\beta$ therapy. Anti-PD-L1 treatment alone or in combination with anti-TGF- $\beta$ induced immune response-related genes in both fibroblasts and immune cells, including multiple chemokines associated with the recruitment of cytotoxic cells. The combination of anti-PD-L1 plus anti-TGF- $\beta$ enhanced the expression of many of these genes over anti-PD-L1 alone, and thus, the combination appears to work together to alter the expression of fibroblasts in a way that may enhance immune infiltration into tumors.

Our scRNA-seq data set comprises almost 30,000 single-cell transcriptomes across four treatment groups. This is a significant community resource for discovery of novel biomarkers and therapeutic targets. Though patient response to PD-1/PD-L1 checkpoint inhibition remains difficult to predict, biomarkers for positive outcomes in anti-PD-L1 therapy include IHC for tumor PD-L1 and tumor mutational burden [31], while biomarkers for anti-TGF- $\beta$ are unknown. Our data suggest that extracellular matrix or chemokine receptor genes could be used to identify patients more likely to achieve immune cell infiltration for anti-PD-L1 and/or antiTGF- $\beta$ therapy. Our work also identified dozens of genes that could be further investigated as targets for cancer therapy. For example, collagen and metalloproteinase genes such as Col1a1, Col1a2, Cthrc1, P3h4, and Mmp2, which were downregulated in fibroblasts, could be targeted therapeutically to encourage immune cell infiltration. Our work also suggested that chemokines, cytokines, and their receptors (e.g., Ccl5, Cxcl10, Cxcl9, $\mathrm{Ccl} 4, \mathrm{Ccl} 3, \mathrm{Ccl} 2, \mathrm{Ccl} 7$ ) were involved in immune cell infiltration and therefore represent attractive therapeutic targets. Such molecules could be blocked or activated by monoclonal antibodies, or attached to tumor-directed antibodies as bifunctional molecules [32]. Though there is extensive research on the involvement of chemokines, cytokines, and their receptors in tumor models [18, 19], our work uniquely identifies molecules specific to antiPD-L1 and/or anti-TGF- $\beta$ therapy, suggesting possibilities for combination therapies. However, all such targets require significant further research. For example, not all chemokines enhance immune responses, and instead, some may actually inhibit anti-tumor immunity [33, 34]. 


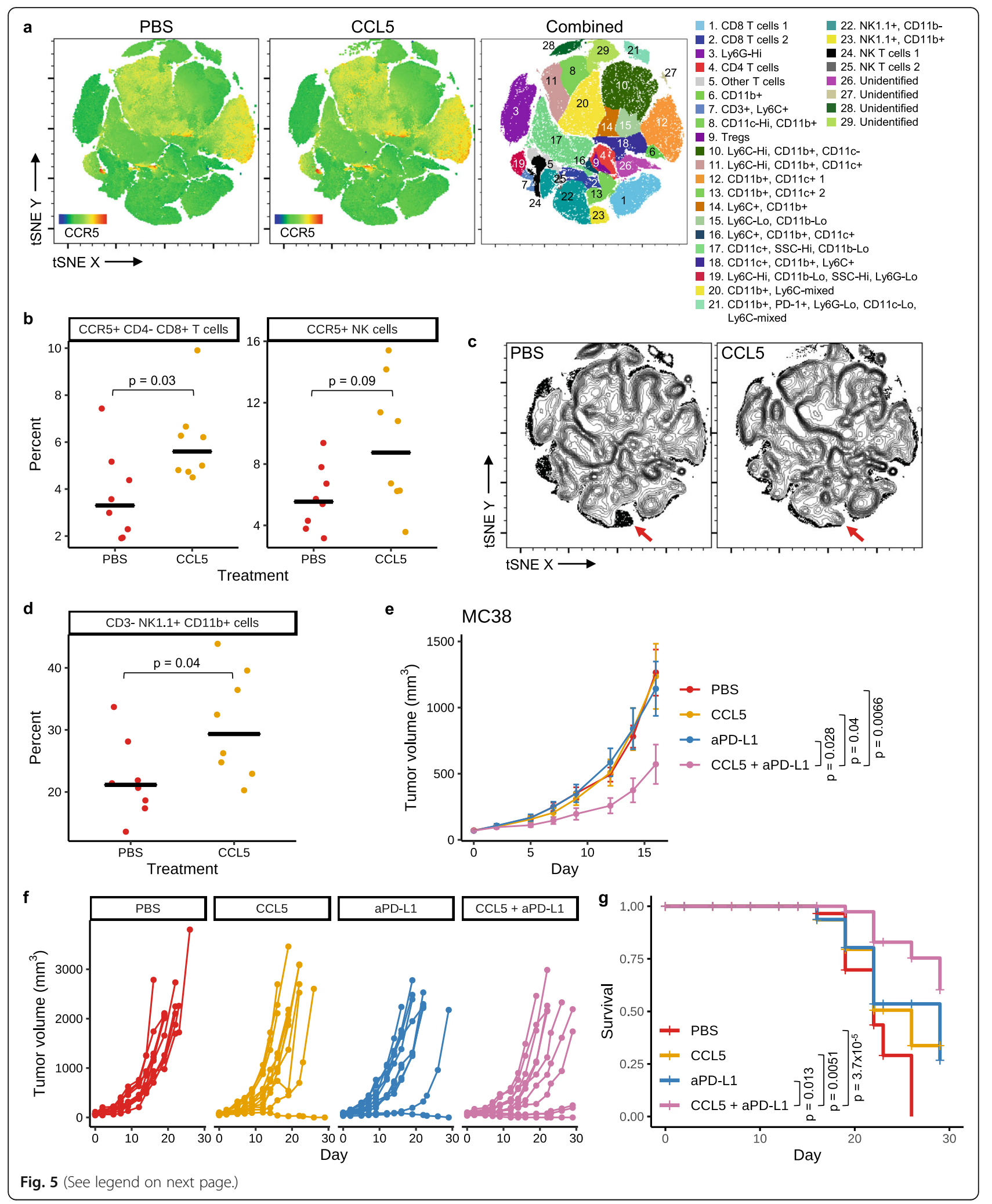


(See figure on previous page.)

Fig. 5 Combination therapy of anti-PD-L1 plus intratumorally administered CCL5 leads to tumor growth inhibition. a-d Mice bearing s.c. MC38 tumors were administered PBS or CCL5 (1 $\mathrm{\mu g} /$ dose) I.T. three times per a week for 5 doses, and on day 12 tumors were harvested and analyzed by flow cytometry. a tSNE plots of flow data showing the expression of CCR5 within PBS control (left) or CCL5-treated tumors (middle), or where populations are colored according to expression of marker genes as indicated in the key (right). Heat maps showing expression of each flow marker are in Additional file 1, Figure S8. b Left: percent of CCR5+ cells within the CD8+ T cell population (live cells, CD4-, CD8+). Right: percent of CCR5+ cells within the NK cell population (live cells, NK1.1+, CD3-). Black cross bars represent median values. $P$ values were determined using Wilcoxon rank sum test. c Density plots (with outlier cells indicated as dots) of populations from the tSNE analysis in a within control or CCL5treated samples. Red arrows indicate CD11 b+ NK cells (population 23 from a). d Frequency of NK cells (live, single cells, NK1.1+ CD3-) that express CD11b as determined by flow cytometry. Black cross bars represent median values. $P$ value was determined using Wilcoxon rank sum test. e-g Mice bearing s.c. MC38 tumors ( $n=12$ per group) were administered PBS (I.T., 3 times a week for 3 weeks), CCL5 (I.T., 1 Mg/animal, 3 times a week for 3 weeks), aPD-L1 (atezolizumab; I.P., 1 mg/kg, twice a week for 3 weeks), or a combination of CCL5 and aPD-L1. e Average tumor volume \pm SEM for each treatment group is shown. $P$ values were determined using Wilcoxon rank sum test, comparing tumor sizes on day 16 . $\mathbf{f}$ Spider plots showing tumor volume for individual mice over time. $\mathbf{g}$ Survival plot for the same study. $P$ values were determined using log-rank test. Non-significant $p$ values are not shown for all panels

Because our scRNA-seq study pooled multiple tumors together for a single experiment, future studies could include performing scRNA-seq on multiple individual tumors to confirm and expand upon the results seen here. In addition, because the dissociated single cells analyzed here were cryopreserved then later thawed prior to scRNA-seq, subsequent studies using freshly prepared cells would be desirable to reduce any potential changes to the original composition of CD45+ and CD45- cells.

The particular case of CCL5 highlights the challenges of understanding and modulating the complex pathways underlying the tumor microenvironment. Specifically, conflicting data exist as to whether the CCL5 pathway should be viewed as a target for activation or a target for inhibition. For example, and consistent with our observations that CCL5 expression in the tumor enhanced anti-PD-L1 therapy, higher CCL5 expression in the tumor has been associated with significantly improved progression-free survival in patients treated with the anti-PD-L1 immunotherapy atezolizumab in multiple clinical trials [35]. Further, CCL5 and its receptor CCR5 are required for productive anti-tumor responses following immunotherapy [36-39]. More specifically, blockade of CCL5 after tumor establishment limited the antitumor effect of CD40 agonism plus immune checkpoint blockade [28]. In contrast, CCL5 enhances homing of Tregs in some tumor models, and the transplantable pancreatic tumor model KPC has been shown to grow less aggressively in mice lacking CCL5 [40-43]. Additionally, CCL5 inhibition has been shown to limit monocyte recruitment and reprograming to immunosuppressive tumor-associated macrophages [44]. Thus, CCL5 may have a different role in controlling the makeup of the tumor microenvironment in developing versus established tumors, especially in the context of immunotherapy. This raises the possibility that combination therapies that deplete Tregs or tumor-associated macrophages may enhance the anti-tumor effect of CCL5 administration in some contexts. Alternatively, therapies that select for antitumorigenic potential of these cell types, such as the effect anti-PD-L1 may have on tumor-associated macrophages, may also enhance the anti-tumor effect of CCL5. Our data showed that intratumoral injection of CCL5 into MC38 tumors might enhance the fraction of $\mathrm{CD} 8+\mathrm{T}$ cells positive for the CCL5 receptor CCR5, could increase intratumoral levels of mature CD11b+ NK cells, and in combination with an otherwise ineffective dose of antiPD-L1, limited tumor progression. Thus, we hypothesize that in the therapeutic setting, exogenous CCL5 primarily recruits cells that are able to participate in an anti-tumor immune response in the presence of anti-PD-L1. Future studies should help to elucidate the specific cell types and mechanisms leading to this therapeutic effect and to determine whether CCL5-based therapies have any potential toxicity advantage over blocking TGF- $\beta$.

\section{Conclusions}

Here we profiled the transcriptional changes within the tumor microenvironment associated with anti-PD-L1 and/or anti-TGF- $\beta$ therapy in a preclinical cancer model at the single-cell level. As has previously been hypothesized, we found that anti-PD-L1 plus anti-TGF- $\beta$ therapy downregulated extracellular matrix genes within fibroblasts, which may enhance the ability of $\mathrm{T}$ cells to traffic within tumors. Furthermore, in multiple cell types, we found that these therapies upregulated genes responsible for recruiting cytotoxic lymphocytes, including many chemokines. Analysis of TCGA data revealed that expression of the chemokine CCL5 within patient tumors was highly associated with lymphocyte infiltration. This led us to test and validate the therapeutic potential of CCL5 treatment plus anti-PD-L1 in the preclinical MC38 tumor model. These data could help identify additional novel targets that could be modulated to overcome resistance to anti-PD-(L)1 therapies. 


\section{Methods}

\section{Murine models}

Murine experiments were approved by the Institutional Animal Care and Use Committees of Explora BioLabs, Crown Bioscience, or Champions Oncology.

Experiments using Hu-PD-L1 KI mice were performed at Crown Bioscience (Taicang Jiangsu Province, China), and mice were obtained from Shanghai Model Organisms Center, Inc. Crown Bioscience acquired MC38 cells from FDCC (The Institutes of Biomedical Sciences (IBS), Fudan University) and authenticated cell identity by SNP analysis. MC38 cells expressing human PD-L1 (Hu-PDL1 MC38) were created by knocking out murine B7h1 (expressing PD-L1) and transgenically expressing human B7H1 driven by the CMV promoter. MC38 and Hu-PDL1 MC38 cells were tested for mycoplasma after cell banking. Two independent in vivo Hu-PD-L1 MC38 tumor experiments were performed. In the first study, 1 $\times 10^{6} \mathrm{Hu}-\mathrm{PD}-\mathrm{L} 1 \mathrm{MC} 38$ were implanted s.c. into the flank of Hu-PD-L1 knock-in C57BL/6 mice (female, 911 weeks old). The mice were randomized into treatment groups ( $n=6$ per group) when average tumor volume reached $90.48 \mathrm{~mm}^{3}$. Dosing was initiated on the same day (day 0). The mice were dosed I.P. biweekly for 3 weeks with PBS, anti-PD-L1 (atezolizumab, Roche, 2 $\mathrm{mg} / \mathrm{kg})$, or a combination of anti-PD-L1 $(2 \mathrm{mg} / \mathrm{kg})$ and anti-TGF- $\beta$ (mouse IgG1 clone 1D11 from BioXCell, 10 $\mathrm{mg} / \mathrm{kg}$ ). Tumor volumes and body weight were measured in a blinded fashion twice a week. Tumor volumes were calculated using the formula: $V=(L \times W \times W) / 2$, where $V$ is tumor volume, $L$ is tumor length (the longest tumor dimension), and $W$ is tumor width (the longest tumor dimension perpendicular to $L$ ). Individual animals were removed from the study as their tumor volumes measured greater than $3000 \mathrm{~mm}^{3}$. All mice that did not have a complete response within the initial 28-day observation period were euthanized, and tumors were collected as formalin-fixed paraffin-embedded (FFPE) blocks. Mice with full tumor regression (tumor volume of $0.00 \mathrm{~mm}^{3}$ ) after 4 weeks were re-challenged on study day 49. Six wildtype C57BL/6 mice (16-18 weeks of age) from Shanghai Lingchang Biotechnology Co., Ltd were used as a naïve control for the re-challenge experiment. Mice for the re-challenge experiment were inoculated s.c. at the opposite flank (left lower flank) with $1 \times 10^{6}$ $\mathrm{Hu}-\mathrm{PD}-\mathrm{L} 1-\mathrm{MC} 38$ cells. Following tumor implantation, tumor volume and body weight were measured twice a week in a blinded manner for an additional 4 weeks.

In the second Hu-PD-L1 MC38 study, $1 \times 10^{6} \mathrm{Hu}-$ PD-L1 MC38 were implanted s.c. into the flank of HuPD-L1 knock-in C57BL/6 mice (female, 9-11 weeks old). The mice were randomized into treatment groups $(n=6$ per group) when average tumor volume reached 99.3 $\mathrm{mm}^{3}$. Dosing was initiated on the same day (day 0 ). The mice were dosed I.P. biweekly for 3 weeks with PBS, anti-PD-L1 (atezolizumab, Roche, $2 \mathrm{mg} / \mathrm{kg}$ ), anti-TGF- $\beta$ (mouse IgG1 clone 1D11 from BioXCell, $10 \mathrm{mg} / \mathrm{kg}$ ), or a combination of anti-PD-L1 $(2 \mathrm{mg} / \mathrm{kg})$ and anti-TGF- $\beta$ $(10 \mathrm{mg} / \mathrm{kg})$. Tumor volumes and body weight were measured in the same manner as in the first study, and individual mice were removed from the study as their tumor volumes measured $>3000 \mathrm{~mm}^{3}$. In both studies, twosided Wilcoxon rank sum test was used to determine if the differences in average tumor volume are statistically significant. Survival analysis was performed using the $\mathrm{R}$ package survminer (version 0.4.5), and $p$ values were determined using log-rank test.

The EMT6 tumor study was performed at Champions Oncology, Inc. Champions Oncology acquired EMT6 cells from ATCC and routinely have STR analysis and pathogen testing, including for mycoplasma, of these cells completed by IDEXX BioAnalytics. For the in vivo EMT6 tumor experiment, $2.5 \times 10^{5}$ EMT6 cells suspended in $0.1 \mathrm{~mL} 1 \times$ PBS were implanted orthotopically in the 4th right mammary fat pad of BALB/c mice (female, 7.5-12 weeks old; Taconic). When tumors reached an average tumor volume of $176.56 \mathrm{~mm}^{3}$ with a range of $15-400 \mathrm{~mm}^{3}$, animals were matched by tumor volume into four blinded groups $(n=12$ mice per group) on day 0 . The four treatment groups were PBS, anti-PD-L1 $(10 \mathrm{mg} / \mathrm{kg}$ for the first dose with each subsequent dose at $5 \mathrm{mg} / \mathrm{kg}$; atezolizumab, Roche), anti-TGF- $\beta$ (10 mg/kg; mouse IgG1 clone 1D11, BioXCell), or a combination of anti-PD-L1 plus anti-TGF- $\beta$. For all treatments, the first dose was administered intravenously (I.V.) on day 0 and the eight subsequent doses were administered I.P. using a dose volume of 5 $\mathrm{mL} / \mathrm{kg}$ three times per a week. Tumor volumes were measured three times weekly by a person blinded to treatment group. Mice were observed for 28 days post initiation of dosing, with individual animals being removed from the study as their tumor volumes measured $>2000 \mathrm{~mm}^{3}$ and collected for FFPE. On day 8 , three representative mice per group were sacrificed, chosen based on day 6 tumor volumes that were similar to the average tumor volume for each treatment group. Whole tumors from the selected mice were sterilely harvested, removing adjacent skin but leaving the exterior surface of the tumor intact to preserve the tumor microenvironment. If there was a large amount of mammary fat attached to the tumor or if the tumor had invaded the adjacent tissue, the tumor was cut away from the tissue and approximately $1-2 \mathrm{~mm}$ of the tissues was kept attached to the tumor, so that the boundary between the tumor and non-tumor tissues was not disturbed. Tumors were placed into MACS Tissue Storage Solution (Miltenyi Biotec) on ice packs and shipped overnight for scRNA-seq processing. 
The CT26 tumor study was performed at Explora BioLabs by GigaGen staff. CT26 cells were obtained from ATCC and expanded to generate a research cell bank, which was used for murine experiments after a representative vial was found to be mycoplasma negative. $5 \times 10^{5}$ CT26 cells suspended in $0.1 \mathrm{~mL} 1 \times \mathrm{PBS}$ were implanted s.c. into the right hind flank of BALB/C mice (female, 8-10 weeks old; Taconic). On day 10 post implantation when tumors were an average tumor volume of $40.01 \mathrm{~mm}^{3}$ (range $25.5-63.89 \mathrm{~mm}^{3}$ ), animals were randomized into seven blinded groups based on tumor volume. Group 1 received PBS I.P. $(n=10)$. Group 2 received anti-PD-L1 I.P. ( $2 \mathrm{mg} / \mathrm{kg}$; atezolizumab, Roche; $n=12$ ). Group 3 received anti-TGF- $\beta$ I.P. $(10 \mathrm{mg} / \mathrm{kg}$; clone 1D11, BioXcell; $n=10)$. Group 4 received anti-PD-L1 I.P. + anti-TGF- $\beta$ I.P. $(n=12)$. Group 5 received anti-PD-L1 I.P. $(2 \mathrm{mg} / \mathrm{kg})+$ PBS I.T. $(n=12)$. Group 6 received anti-TGF- $\beta$ I.T. $(40 \mu \mathrm{g} / \mathrm{dose} ; n=6)$. Group 7 received anti-PD-L1 I.P. $(2 \mathrm{mg} / \mathrm{kg})+$ anti-TGF$\beta$ I.T. $(40 \mu \mathrm{g} /$ dose $)(n=12)$. Starting on day 11 , mice were treated two times a week for 3 weeks. Tumors were measured three times weekly. Mice were observed for 35 days post implantation, with individual animals being removed from the study as their tumor volumes measured greater than $2000 \mathrm{~mm}^{3}$.

The MC38 tumor study was performed at Champions Oncology, Inc. $5 \times 10^{5}$ MC38 cells suspended in $100 \mu \mathrm{l}$ of PBS were injected s.c. into the left flank of C57BL/6 mice (female, 7.5-12 weeks old; Taconic). When tumors reached an average tumor volume of $71.1 \mathrm{~mm}^{3}$ with a range of $32-113 \mathrm{~mm}^{3}$, animals were randomized into four blinded groups ( $n=12$ mice per group) on day 0 . The treatment groups were PBS, recombinant human CCL5 (R\&D systems, 278-RN-050/CF), PBS plus antiPD-L1 (atezolizumab, Roche), and CCL5 plus anti-PDL1. PBS and CCL5 (1 $\mu \mathrm{g} / \mathrm{animal})$ were administered I.T., three times a week for 3 weeks. Anti-PD-L1 $(1 \mathrm{mg} / \mathrm{kg})$ was administered I.P., twice a week for 3 weeks. Dosing started on day 1 . Tumor volumes were measured three times weekly. Mice were observed for 28 days, with individual animals being removed from the study as their tumor volumes measured $>2000 \mathrm{~mm}^{3}$, and the tumors were collected as FFPE. Two-sided Wilcoxon rank sum test was used to determine statistical significance in difference in average tumor volume.

\section{Immunohistochemistry}

All immunohistochemistry (IHC) was performed by Allele Biotechnology. Four-micrometer sections were cut and placed on glass slides for CD3 antibody staining (clone SP7, Abcam). An IHC score was blindly assigned to each stained tumor section based on the observed density of CD3+ cells across the entire slide (1 to 5, where $1=<5 \%, 2=5-15 \%, 3=15-30 \%, 4=30-50 \%, 5$
$=>50 \%)$. Statistical significance in the difference in CD3+ cell score was determined using Wilcoxon rank sum test.

\section{Single-cell RNA sequencing}

EMT6 mammary fat pad tumors and the neighboring tissue were collected from mice such that the entire tumor and the interface between tumor and normal tissue was preserved, and the tissue was stored overnight at $4{ }^{\circ} \mathrm{C}$ in MACS Tissue Storage Solution (Miltenyi Biotec). Because the tumors were small at the time of harvest, multiple tumors for each treatment group were pooled to ensure that sufficient viable cells remained after all of the following steps. Tumor tissue was minced and dissociated using the mouse Tumor Dissociation Kit (Miltenyi Biotec) and gentleMACS Octo Dissociator with Heaters (Miltenyi Biotec). The "37C m_TDK_2" and "m_imptumor_01" programs were used for the primary and secondary gentle MACS dissociation programs. Tumor suspensions were filtered through a 70$\mu \mathrm{m}$ MACS Smart Strainers (Miltenyi Biotec) and washed with PBS containing $0.5 \%$ BSA and $2 \mathrm{mM}$ EDTA. Cells were pelleted by centrifugation at $500 \times g$ for $7 \mathrm{~min}$. A red blood cell lysis step was performed by incubating cells for $2 \mathrm{~min}$ with $1 \times$ Red Blood Cell Lysis Solution (Miltenyi Biotec). Cells were washed again and then cell viability was measured using the Nexcelom Cellometer K2 Fluorescent Viability Cell Counter and the ViaStain AOPI Staining Solution (Nexcelom). Single-cell suspensions were then cryopreserved using CryoStor CS10 solution (STEMCELL Technologies) and stored in liquid nitrogen.

Cells were thawed and FACS sorted for DAPICD45+ (live, hematopoietic cells) and DAPI- CD45(live, non-hematopoietic cells) populations. Briefly, cells were thawed in a $37{ }^{\circ} \mathrm{C}$ water bath, resuspended in 10 $\mathrm{mL}$ of RPMI (Gibco) with 10\% FBS (Gibco), centrifuged at $500 \times g$ for $5 \mathrm{~min}$, washed in PBS containing $0.5 \% \mathrm{BSA}$ and $2 \mathrm{mM}$ EDTA, and counted using the Nexcelom Cellometer. Approximately $10 \times 10^{6}$ cells were transferred to a new tube, washed in PBS with $0.5 \%$ BSA and $2 \mathrm{mM}$ EDTA, and centrifuged at $500 \times g$ for $5 \mathrm{~min}$. The liquid was poured off from the cells (leaving approximately $100 \mu \mathrm{l}$ in each tube) and $5 \mu \mathrm{l}$ of mouse $\mathrm{Fc}$ Blocking Reagent (rat anti-Mouse CD16/CD32; BD Biosciences) was added to samples and incubated for $5 \mathrm{~min}$ at $4{ }^{\circ} \mathrm{C} .162 .5 \mu \mathrm{l}$ of Brilliant Stain Buffer (BD Biosciences) containing $12.5 \mu \mathrm{l}$ of mouse CD45-PE clone 30-F11 (BioLegend) was added to cells and incubated on ice for 30 min protected from light. Cells were washed, resuspended in $1 \times$ DAPI solution (in PBS plus $0.5 \%$ BSA and $2 \mathrm{mM}$ EDTA; BioLegend) at $5 \times 10^{6}$ cells $/ \mathrm{ml}$, and filtered into $5 \mathrm{~mL}$ FACS tubes with cell-strainer caps (Falcon). Cells were sorted using the BD FACSMelody using 
the $100 \mu \mathrm{m}$ nozzle. Mouse splenocytes were used as a positive control to determine the voltage needed to identify CD45+ immune cells in the initial side scatter area and forward scatter area gate that would be used for tumor cell suspensions. Singlet and DAPI- (live cell) gates were then applied. Stained single-cell suspensions from EMT6 tumors were then sorted and approximately $1 \times 10^{5}$ live CD $45+$ and $1 \times 10^{5}$ live CD45- cells were collected at $4{ }^{\circ} \mathrm{C}$. Cells were washed in RPMI with $10 \%$ FBS, centrifuged at $500 \times g$ for $5 \mathrm{~min}$, and counted as described above. Cells were resuspended in RPMI with $10 \%$ FBS at $7 \times 10^{5}$ cells $/ \mathrm{ml}$ and were placed on ice. CD45+ and CD45- sorted cells from each of the four treatment groups were run on the 10x Genomics Chromium device using the Single Cell 3' Reagent Kit v3 (work performed at SeqMatic). Libraries were sequenced at SeqMatic with an Illumina NovaSeq SP 100 cycle kit (28 bp for read 1, $91 \mathrm{bp}$ for read 2).

\section{Single-cell RNA-seq data analysis}

Raw sequencing FASTQ files were processed using the Cell Ranger (version 3.1.0) analysis pipeline. Briefly, alignment and filtering of sequencing reads, barcode counting, and UMI counting were performed using the cellranger count command. The reads were aligned to the Mus musculus reference genome assembly GRCm38 (mm10). The outputs of cellranger count for all samples were aggregated using the command cellranger aggr, normalizing the runs to the same sequencing depth. Secondary analyses were performed using the Seurat package (version 3.2.3) in $\mathrm{R}$ (version 4.0.3) [45]. First, we performed quality control by removing cells with fewer than 200 or more than 5000 expressed genes, as low and high number of gene counts may indicate low quality cells and cell multiplets, respectively. We also removed cells with more than $10 \%$ mitochondrial gene expression (Additional file 1, Figure S3). This resulted in 27,460 cells, including 17,665 CD45+ and 9795 CD45- cells. We used sctransform to normalize the expression data and to regress out mitochondrial mapping percentage as a confounding source of variation [46]. We performed principal component analysis using the RunPCA function in Seurat. Principal components 1 to 30 were provided as an input for non-linear dimensionality reduction via Uniform Manifold Approximation and Projection (UMAP) using the RunUMAP function.

For cell type annotation, we used the $\mathrm{R}$ package SingleR, which leveraged reference transcriptomic datasets of pure cell types to infer the cell of origin of each of the single cells independently [47]. The ImmGen (www. immgen.org) reference dataset was used. The cells were first annotated using the main cell type labels generated by SingleR (note: the initial data for T cells, NKT, and Tgd were highly mixed together, making it hard to distinguish each as separate cell types, and thus the data for these cell types were combined and reported as $\mathrm{T}$ cells for all subsequent analyses). Since the ImmGen reference did not include tumor cell as a cell type, we needed to manually discern tumor cells from host nonimmune cells within the CD45- cells. We therefore reanalyzed the CD45- cells using RunUMAP, followed by clustering using the FindNeighbors and FindClusters functions in Seurat. These functions applied shared nearest neighbor (SNN)-based clustering on the scRNAseq data, identifying 15 clusters of cells. Cells in cluster 7 were annotated as fibroblasts based on expression of fibroblast marker gene Fap. Cells previously annotated by SingleR as endothelial cells or epithelial cells were assigned their original SingleR labels. All other CD45cells were annotated as tumor cells. To validate the annotations, we analyzed copy number alterations for the CD45- cells using InferCNV [48], using epithelial and endothelial cells as reference cells.

\section{Differential gene expression and functional enrichment analysis}

We performed differential gene expression analysis within each cell type using the FindMarkers function in Seurat. We compared each treatment group to the PBS control, using Wilcoxon rank sum test to identify differentially expressed (DE) genes (log fold change $>0.25$, adjusted $p$ value $<0.05$ ) between the two groups of cells. We also compared the anti-PD-L1 plus anti-TGF- $\beta$ treatment group to the single treatment groups. We note that because multiple tumors were pooled for each treatment group for the scRNA-seq analysis, it is possible that some of the gene expression differences observed could be skewed by a single tumor sample. We used clusterProfiler (version 3.18.0) [49] to perform functional enrichment analysis. For each treatment group, we generated foreground gene lists containing genes upregulated or downregulated in the cell type of interest. These gene lists were tested against background gene lists containing all expressed genes (expressed in at least 1 cell) in the cell type of interest. The enrichment analysis was performed using the enrich $G O$ function in clusterProfiler using the org.Mm.eg.db database and the gene ontology (GO) categories biological process (BP), molecular function (MF), and cellular component (CC). Multiple testing correction was performed using the Benjamini and Hochberg method. The enriched pathways were visualized as gene concept networks using the cnetplot function of the enrichplot package (version 1.10.0) [50].

\section{Association of chemokine expression and immune cell abundance in TCGA}

We inferred intratumoral immune cell abundance using either an image-based approach or a gene signature-based 
approach. For the image-based approach, we downloaded 4612 published TIL scores estimated from TCGA H\&E images [23, 24]. Briefly, Saltz et al. developed deep learning-based image recognition to classify and quantify lymphocytes on H\&E diagnostic images from 13 TCGA cancer types (LUAD, BRCA, PAAD, COAD, LUSC, PRAD, UCEC, READ, BLCA, STAD, CESC, SKCM, and UVM). The deep learning model training process was repeated until pathologists judged that the lymphocyte classification was adequate. The TIL scores were reported as "TIL Regional Fraction" in Table S1 by Thorsson et al. [24]. For the gene signature-based approach, we downloaded pre-calculated xCell immune cell scores for 9358 TCGA samples [25] and filtered for immune cell types of interest: $\mathrm{CD} 8+\mathrm{T}$ cells, dendritic cells $(\mathrm{aDC})$, NK cells, Tregs, and M2 macrophages.

For TCGA tumor gene expression data, we downloaded RNA-seq quantifications (in fragments per kilobase of transcript per million mapped reads, FPKM) from the TCGA portal [51] for 32 cancer types (ACC, BLCA, BRCA, CESC, CHOL, COAD, DLBC, ESCA, GBM, HNSC, KICH, KIRC, KIRP, LGG, LIHC, LUAD, LUSC, MESO, OV, PAAD, PCPG, PRAD, READ, SARC, SKCM, STAD, TGCT, THCA, THYM, UCEC, UCS, UVM). We filtered for chemokine genes by retaining only expression data for genes whose names started with CXC, CCL, CX3C, or XC. Within each cancer type, genes that were not or lowly expressed (median expression across all samples $<0.01$ FPKM) were excluded from further analysis.

For each TCGA cancer type, the chemokine gene expression data frame was combined with either the image-based or the gene signature-based immune score data frame. Only samples with data present in both data frames were analyzed. A pseudo count of 0.01 was added to the expression and immune scores, followed by $\log _{2}$ and $z$-score transformation, in preparation for linear regression analysis. We performed linear regression analysis for each chemokine gene using immune score as the dependent variable and chemokine gene expression as the independent variable. $P$ values were adjusted for multiple testing using the Benjamini and Hochberg method. Chemokines significantly associated with immune scores were further ranked by the sum of their regression coefficients across different cancer types. The results were visualized as heatmaps using ggplot2 (version 3.3.3) [52]. All data manipulations and analyses were performed in $\mathrm{R}$ 4.0.3.

\section{Flow cytometry analysis}

An additional cohort of C57BL/6 mice bearing MC38 tumors were treated with 5 doses I.T. PBS or CCL5 $(1 \mu \mathrm{g} /$ animal $)$ on days $1,3,5,8$, and 10 as indicated in the murine models section above. Tumors were harvested on day 12, cut into small pieces approximately of $2-4 \mathrm{~mm}$, and dissociated using the Miltenyi Biotec MACS Tumor Dissociation Kit according to manufacturer instructions (all performed by Champions Oncology). Dissociated cell suspensions were filtered through a 70- $\mu \mathrm{m}$ strainer with $10 \mathrm{~mL}$ RPMI 1640 (Thermo Fisher Scientific) and centrifuged at $300 \times g$ for $7 \mathrm{~min}$. Cells were resuspended in MACS Quant running buffer (Miltenyi Biotec), and red blood cells were lysed using RBC Pharm Lyse (BD Biosciences). Cells were pelleted, washed, and resuspended with MACs buffer. Cells were then put into 96-well plates and stained with fluorescently labelled antibodies and FVS780 viability stain. Cells were analyzed using BD FACSymphony. FlowJo 10 was used to generate tSNE plots of cells pre-gated based on live, single cells and taking into account data from side scatter, CD11b (BUV395, clone M1/70, BD Biosciences), CD4 (BUV496, clone RM4-5, BD Biosciences), CD8 (9BUV661, clone 53-6.7, BD Biosciences), CD3 (BUV805, clone 17A2, BD Biosciences), CD11c (BV421, clone N418, BD Biosciences), Ly6G (BV605, clone 1A8, BD Biosciences), CD44 (BV786, clone IM7, BD Biosciences), Ly6C (AF488, clone HK1.4, BioLegend), PD-1 (BB700, clone j43, BD Biosciences), FOXP3 (PE, clone FJK-16S, Fisher Scientific), NK1.1 (PECy7, clone PK136, BioLegend), and CCR5 (CD195; APC, Clone 2D7, BD Biosciences) staining. Populations were named based on expression of the indicated marker(s).

\section{Supplementary Information}

The online version contains supplementary material available at https://doi. org/10.1186/s12915-021-01034-z.

Additional file 1. Supplementary figures. Figure S1. Anti-PD-L1 plus anti-TGF- $\beta$ in the murine tumor model HuPD-L1-MC38. Figure S2. AntiPD-L1 plus anti-TGF- $\beta$ in the murine tumor model CT26. Figure S3. Quality control of single cell RNA-seq of anti-PD-L1 \pm anti-TGF- $\beta$ treated EMT6 tumor-bearing mice. Figure S4. CD45-cell analysis and InferCNV of antiPD-L1 1 anti-TGF- $\beta$ treated EMT6 tumor-bearing mice. Figure S5. scRNAseq marker gene expression. Figure S6. scRNA-seq cell type composition and chemokine expression. Figure S7. Association of chemokine gene expression with inferred immune cell infiltration in TCGA. Figure S8. Flow cytometry-based expression of key genes in tSNE identified populations.

Additional file 2. Supplementary tables. Table S1. Differentially expressed (DE) genes determined from the EMT6 single cell RNA-seq experiment. Table S2. Differentially expressed (DE) genes in fibroblasts. Table S3. Differentially expressed (DE) genes in macrophages.

\section{Acknowledgements}

The authors appreciate thoughtful discussions from Matthew Spindler, Kyle Carter, Jan Simons, Ellen Wagner, Nicholas Wayham, and Jasmeen Saini (GigaGen). The authors also thank Penny Chen and Michael Boice (Crown Bioscience) and Mary Topalovski and Monika Buczek (Champions Oncology) for assistance with efficacy studies, and Abbas Hussain and Anthony Wong (Allele Biotechnology) for assistance with $\mathrm{IHC}$.

\section{Authors' contributions}

Y.W.L., A.S.A., and E.L.S. conceived and designed the study; Y.W.L., G.L.C., and S.K.S. performed the experiments; Y.W.L., S.K.S., A.S.A., and E.L.S. analyzed the 
data; Y.W.L., D.S.J., A.S.A., and E.L.S. wrote and edited the manuscript; D.S.J., A.S.A., and E.L.S. provided supervision and project administration; D.S.J. acquired the funding. All authors read and approved the final manuscript.

\section{Funding}

This study was funded in part by the NIH grant NCI R44CA187852 to D.S.J.

\section{Availability of data and materials}

Single-cell RNA-seq fastq sequence files are deposited at the Sequence Read Archive under BioProject ID PRJNA615238 [53]. The flow data is available upon request from the corresponding authors. All other data is provided in the published article and its supplementary files.

\section{Declarations}

\section{Ethics approval and consent to participate}

Not applicable.

\section{Consent for publication}

Not applicable.

\section{Competing interests}

All authors are salaried employees of GigaGen, a subsidiary of Grifols. GigaGen provided research funds for described studies. Y.W.L., G.L.C., D.S.J., A.S.A., and E.L.S. are inventors on provisional patent application 63/023,790 (assigned to GigaGen) describing therapeutic modalities for cancer that incorporate chemokines such as CCL5.

\section{Received: 29 October 2020 Accepted: 21 April 2021}

Published online: 25 May 2021

\section{References}

1. Tumeh PC, Harview CL, Yearley JH, Shintaku IP, Taylor EJM, Robert L, et al. PD-1 blockade induces responses by inhibiting adaptive immune resistance. Nature. 2014;515(7528):568-71. https://doi.org/10.1038/nature13954.

2. Chen DS, Mellman I. Elements of cancer immunity and the cancerimmune set point. Nature. 2017;541(7637):321-30. https://doi.org/10.103 8/nature21349.

3. Mariathasan S, Turley SJ, Nickles D, Castiglioni A, Yuen K, Wang Y, et al. TGF $\beta$ attenuates tumour response to PD-L1 blockade by contributing to exclusion of T cells. Nature. 2018;554:544-8.

4. Routh ED, Pullikuth AK, Jin G, Chifman J, Chou JW, D'Agostino RB Jr, et al. Transcriptomic features of $\mathrm{T}$ cell-barren tumors are conserved across diverse tumor types. Front. Immunol. 2020;11:991-19.

5. Tauriello DVF, Palomo-Ponce S, Stork D, Berenguer-Llergo A, Badia-Ramentol $J$, Iglesias M, et al. TGF $\beta$ drives immune evasion in genetically reconstituted colon cancer metastasis. Nature. 2018;554:538-43.

6. Sow HS, Ren J, Camps M, Ossendorp F, Dijke ten P. Combined inhibition of TGF- $\beta$ signaling and the PD-L1 immune checkpoint is differentially effective in tumor models. Cells. 2019;8:320-314, 4, DOl: https://doi.org/10.3390/ cells8040320.

7. Principe DR, Park A, Dorman MJ, Kumar S, Viswakarma N, Rubin J, et al. TGF $\beta$ blockade augments PD-1 inhibition to promote T-cell-mediated regression of pancreatic cancer. Mol. Cancer Ther. 2019;18:613-20.

8. Lan Y, Zhang D, Xu C, Hance KW, Marelli B, Qi J, et al. Enhanced preclinical antitumor activity of M7824, a bifunctional fusion protein simultaneously targeting PD-L1 and TGF- $\beta$. Sci Transl Med. 2018;10:eaan5488.

9. Holmgaard RB, Schaer DA, Li Y, Castaneda SP, Murphy MY, Xu X, et al. Targeting the TGF $\beta$ pathway with galunisertib, a TGF $\beta R I$ small molecule inhibitor, promotes anti-tumor immunity leading to durable, complete responses, as monotherapy and in combination with checkpoint blockade. J ImmunoTherapy Cancer. 2018;6:47.

10. Dodagatta-Marri E, Meyer DS, Reeves MQ, Paniagua R, To MD, Binnewies M, et al. a-PD-1 therapy elevates Treg/Th balance and increases tumor cell pSmad3 that are both targeted by a-TGF $\beta$ antibody to promote durable rejection and immunity in squamous cell carcinomas. J ImmunoTherapy Cancer. 2019:7:62

11. Mitra MS, Lancaster K, Adedeji AO, Palanisamy GS, Dave RA, Zhong F, et al. A potent pan-TGF $\beta$ neutralizing monoclonal antibody elicits cardiovascular toxicity in mice and cynomolgus monkeys. Toxicol. Sci. 2020;175:24-34.
12. Martin CJ, Datta A, Littlefield C, Kalra A, Chapron C, Wawersik S, et al. Selective inhibition of TGF $\beta 1$ activation overcomes primary resistance to checkpoint blockade therapy by altering tumor immune landscape. Sci Transl Med. 2020;12:eaay8456.

13. Lind H, Gameiro SR, Jochems C, Donahue RN, Strauss J, Gulley JL, et al. Dual targeting of TGF- $\beta$ and PD-L1 via a bifunctional anti-PD-L1/TGF- $\beta R$ Il agent: status of preclinical and clinical advances. J Immuno Therapy Cancer. 2020; 8(1):e000433-e1426519. https://doi.org/10.1136/jitc-2019-000433.

14. Zhang Y, Morgan R, Chen C, Cai Y, Clark E, Khan WN, et al. Mammarytumor-educated $B$ cells acquire LAP/TGF- $B$ and PD-L1 expression and suppress anti-tumor immune responses. Int Immunol. 2016;28(9):423-33. https://doi.org/10.1093/intimm/dxw007.

15. Heng TSP, Painter MW, Consortium IGP. The Immunological Genome Project: networks of gene expression in immune cells. Nat. Immunol. 2008; 9(10):1091-4. https://doi.org/10.1038/ni1008-1091.

16. Gubin MM, Esaulova E, Ward JP, Malkova ON, Runci D, Wong P, et al. Highdimensional analysis delineates myeloid and lymphoid compartment remodeling during successful immune-checkpoint cancer therapy. Cell. 2018;175:1014-9.

17. Xiong H, Mittman S, Rodriguez R, Moskalenko M, Pacheco-Sanchez P, Yang $Y$, et al. Anti-PD-L1 treatment results in functional remodeling of the macrophage compartment. Cancer Research. 2019;79(7):1493-506. https:// doi.org/10.1158/0008-5472.CAN-18-3208.

18. Vilgelm AE, Richmond $A$. Chemokines modulate immune surveillance in tumorigenesis, metastasis, and response to immunotherapy. Front. Immunol. 2019;10:705-14.

19. Nagarsheth N, Wicha MS, Zou W. Chemokines in the cancer microenvironment and their relevance in cancer immunotherapy. Nature Reviews Immunol. 2017;17(9):559-72. https://doi.org/10.1038/nri.2017.49.

20. Rosenberg JE, Hoffman-Censits J, Powles T, van der Heijden MS, Balar AV, Necchi A, et al. Atezolizumab in patients with locally advanced and metastatic urothelial carcinoma who have progressed following treatment with platinum-based chemotherapy: a single-arm, multicentre, phase 2 trial. Lancet. 2016;387(10031):1909-20. https://doi.org/10.1016/S0140-6736(1 6)00561-4.

21. Lim YW, Chen-Harris H, Mayba O, Lianoglou S, Wuster A, Bhangale T, et al. Germline genetic polymorphisms influence tumor gene expression and immune cell infiltration. Proc. Natl. Acad. Sci. U.S.A. 2018;115(50):E11701-10. https://doi.org/10.1073/pnas.1804506115.

22. Chow MT, Ozga AJ, Servis RL, Frederick DT, Lo JA, Fisher DE, et al. Intratumoral activity of the CXCR3 chemokine system is required for the efficacy of anti-PD-1 therapy. Immunity. 2019;50:1498-512 e5.

23. Saltz J, Gupta R, Hou L, Kurc T, Singh P, Nguyen V, et al. Spatial organization and molecular correlation of tumor-infiltrating lymphocytes using deep learning on pathology images. Cell Rep. 2018;23(1):181-7. https://doi.org/1 0.1016/j.celrep.2018.03.086.

24. Thorsson V, Gibbs DL, Brown SD, Wolf D, Bortone DS, Ou Yang T-H, et al. The immune landscape of cancer. Immunity. 2018;48(4):812-4. https://doi. org/10.1016/j.immuni.2018.03.023.

25. Aran D, Hu Z, Butte AJ. xCell: digitally portraying the tissue cellular heterogeneity landscape. Genome Biol. 2017;18(1):220. Available from: https://xcell.ucsf.edu/. https://doi.org/10.1186/s13059-017-1349-1.

26. Gonzalez-Martin A, Gomez L, Lustgarten J, Mira E, Manes S. Maximal T cellmediated antitumor responses rely upon CCR5 expression in both CD4+ and CD8+ T cells. Cancer Research. 2011;71(16):5455-66. https://doi.org/1 0.1158/0008-5472.CAN-11-1687.

27. Böttcher JP, Bonavita E, Chakravarty P, Blees H, Cabeza-Cabrerizo M, Sammicheli S, et al. NK Cells stimulate recruitment of $\mathrm{CDC1}$ into the tumor microenvironment promoting cancer immune control. Cell. 2018;172:1022-37 e14.

28. Huffman AP, Lin JH, Kim SI, Byrne KT, Vonderheide RH. CCL5 mediates CD40-driven CD4+ T-cell tumor infiltration and immunity. JCI Insight. 2020; 5(10). https://doi.org/10.1172/jci.insight.137263.

29. Chiossone L, Chaix J, Fuseri N, Roth C, Vivier E, Walzer T. Maturation of mouse NK cells is a 4-stage developmental program. Blood. 2009;113(22): 5488-96. https://doi.org/10.1182/blood-2008-10-187179.

30. Maghazachi AA. Role of chemokines in the biology of natural killer cells. The chemokine system in experimental and clinical hematology. Berlin: Springer Berlin Heidelberg; 2010. p. 37-58.

31. Gibney GT, Weiner LM, Atkins MB. Predictive biomarkers for checkpoint inhibitor-based immunotherapy. Lancet Oncol. 2016;17(12):e542-51. https:// doi.org/10.1016/S1470-2045(16)30406-5. 
32. Neri D. Antibody-cytokine fusions: versatile products for the modulation of anticancer immunity. Cancer Immunol Res. 2019;7:348-54.

33. Li J, Byrne KT, Yan F, Yamazoe T, Chen Z, Baslan T, et al. Tumor cell-intrinsic factors underlie heterogeneity of immune cell infiltration and response to immunotherapy. Immuni. 2018;49:178-93 e7.

34. Hugo W, Zaretsky JM, Sun L, Song C, Moreno BH, Hu-Lieskovan S, et al. Genomic and transcriptomic features of response to anti-PD-1 therapy in metastatic melanoma. Cell. 2016;165:35-44.

35. WU TD, Madireddi S, de Almeida PE, Banchereau R, Chen Y-JJ, Chitre AS, et al. Peripheral T cell expansion predicts tumour infiltration and clinical response. Nature. 2020;579:274-8.

36. Dangaj D, Bruand M, Grimm AJ, Ronet C, Barras D, Duttagupta PA, et al. Cooperation between constitutive and inducible chemokines enables $T$ cell engraftment and immune attack in solid tumors. Cancer Cell. 2019;35:885900 e10.

37. Araujo JM, Gomez AC, Aguilar A, Salgado R, Balko JM, Bravo L, et al. Effect of CCL5 expression in the recruitment of immune cells in triple negative breast cancer. Scientific Reports. 2018;8:4899.

38. Nesbeth Y, Scarlett U, Cubillos-Ruiz J, Martinez D, Engle X, Turk MJ, et al. CCL5-mediated endogenous antitumor immunity elicited by adoptively transferred lymphocytes and dendritic cell depletion. Cancer Research. 2009; 69(15):6331-8. https://doi.org/10.1158/0008-5472.CAN-08-4329.

39. Nesbeth YC, Martinez DG, Toraya S, Scarlett UK, Cubillos-Ruiz JR, Rutkowski MR, et al. CD4 + T cells elicit host immune responses to MHC Class II ovarian cancer through CCL5 secretion and CD40-mediated licensing of dendritic cells. J Immunol. 2010;184(10):5654-62. https://doi.org/10.4049/ jimmunol.0903247.

40. Tan MCB, Goedegebuure PS, Belt BA, Flaherty B, Sankpal N, Gillanders WE, et al. Disruption of CCR5-dependent homing of regulatory $T$ cells inhibits tumor growth in a murine model of pancreatic cancer. J Immunol. 2009; 182:1746-55.

41. Wang X, Lang M, Zhao T, Feng X, Zheng C, Huang C, et al. Cancer-FOXP3 directly activated CCL5 to recruit FOXP3 + Treg cells in pancreatic ductal adenocarcinoma. Oncogene. 2017;36:3048-58.

42. Chang L-Y, Lin Y-C, Mahalingam J, Huang C-T, Chen T-W, Kang C-W, et al. Tumor-derived chemokine CCL 5 enhances TGF- $\beta$-mediated killing of CD8(+) T cells in colon cancer by T-regulatory cells. Cancer Research. 2012;72(5): 1092-102. https://doi.org/10.1158/0008-5472.CAN-11-2493.

43. Aldinucci D, Colombatti A. The inflammatory chemokine CCL5 and cancer progression. Mediators Inflammation. 2014;2014:1-12. https://doi.org/10.11 55/2014/292376

44. Nie Y, Huang H, Guo M, Chen J, Wu W, Li W, et al. Breast phyllodes tumors recruit and repolarize tumor-associated macrophages via secreting CCL5 to promote malignant progression, which can be inhibited by CCR5 inhibition therapy. Clin Cancer Res. 2019;25:3873-86.

45. Stuart T, Butler A, Hoffman P, Hafemeister C, Papalexi E, Mauck WM, et al. Comprehensive integration of single-cell data. Cell. 2019;177:1888-902 e21.

46. Hafemeister C, Satija R. Normalization and variance stabilization of singlecell RNA-seq data using regularized negative binomial regression. Genome Biol. 2019;20(1):296-15. https://doi.org/10.1186/s13059-019-1874-1.

47. Aran D, Looney AP, Liu L, Wu E, Fong V, Hsu A, et al. Reference-based analysis of lung single-cell sequencing reveals a transitional profibrotic macrophage. Nat. Immunol. 2019;20(2):163-72. https://doi.org/10.1038/s41 590-018-0276-y.

48. Tickle T, TI GC, Brown M, Haas B. inferCNV of the Trinity CTAT Project. 2019. Available from: https://github.com/broadinstitute/inferCNV. Accessed 4 Nov 242019.

49. Yu G, Wang L-G, Han Y, He Q-Y. clusterProfiler: an R package for comparing biological themes among gene clusters. OMICS J Integr Biol. 2012;16(5):2847. https://doi.org/10.1089/omi.2011.0118.

50. Yu G. enrichplot: Visualization of functional enrichment result. 1st ed; 2019. Available from: https://github.com/GuangchuangYu/enrichplot. Accessed 8 Jan 2021.

51. Genomic Data Commons Data Portal, The Cancer Genome Atlas Program. Available from: https://portal.gdc.cancer.gov. Accessed 25 Apr 2018.

52. Wickham H. ggplot2: elegant graphics for data analysis. New York: SpringerVerlag; 2016. Available from: https://ggplot2.tidyverse.org. Accessed 6 Jan 2021.

53. Single cell RNA sequencing of tumors from mice treated with anti-PD-L1 and/or anti-TGFb. NCBI Sequence Read Archive https://www.ncbi.nlm.nih. gov/sra/?term=PRJNA615238 (2021).

\section{Publisher's Note}

Springer Nature remains neutral with regard to jurisdictional claims in published maps and institutional affiliations.
Ready to submit your research? Choose BMC and benefit from:

- fast, convenient online submission

- thorough peer review by experienced researchers in your field

- rapid publication on acceptance

- support for research data, including large and complex data types

- gold Open Access which fosters wider collaboration and increased citations

- maximum visibility for your research: over $100 \mathrm{M}$ website views per year

At BMC, research is always in progress.

Learn more biomedcentral.com/submissions 